McGill/92-17.

April 16, 1992.

\title{
Virtual bremsstrahlung from pions and quarks in thermalized hadronic matter
}

\author{
K. Haglin, C. Gale and V. Emel'yanov \\ Physics Dept., McGill University, Montréal, P.Q., H3A 2T8, Canada
}

\begin{abstract}
A soft photon approximation is used to calculate the rates of lepton pair production through virtual bremsstrahlung from both pions and quarks. Standard assumptions about the evolution of a nuclear system under collision allow pion and quark driven total production to be calculated. Comparisons are made with Dalitz decay of light mesons. These mechanisms are expected to be significant contributors to the soft dilepton mass spectra one might observe in heavy ion collisions at RHIC and LHC energies.
\end{abstract}

Thermal photons and dileptons were among the first tools proposed for the observation of thermalized nuclear matter [ []]. Leptons and photons have small cross sections with the nuclear medium, so they will escape without much further interaction from a region of dense hadronic matter. This allows them, at least in principle, to carry information about the temperature of the primordial state, which may be a thermalized hadronic gas or a quarkgluon plasma (QGP). We shall consider here dilepton production from each, concentrating in particular on electron-positron pairs. The dilepton invariant mass naturally subdivides into four distinct domains. For masses $M$ above the $J / \psi$ peak, the spectrum is expected to be dominated by Drell-Yan production [8]. The spectrum around $1 \mathrm{GeV}$ is dominated by $\rho, \omega$ and $\phi$ decays. Dileptons with invariant masses in the $1.5-3.0 \mathrm{GeV}$ region seem to be mostly thermal [3]. Competing sources in this mass region are $D \bar{D}$ decays 沺, Drell-Yan processes, and pre-equilibrium emission [5,6]. The last region of invariant mass $\left(M<m_{\rho}\right)$ is a region of soft lepton production [3]. It is expected that below the $\rho$ mass a non-perturbative QGPinduced dilepton radiation may appear. There are many sources of dileptons with invariant masses less than $m_{\rho}$. For instance, there are $\pi^{0}$ and $\eta$ Dalitz decays, $\pi^{+} \pi^{-}$annihilation from a thermalized pion gas, virtual bremsstrahlung with subsequent decay from pions and quarks, and finally, quark-antiquark annihilation in a QGP. Recent calculations [7] have 
shown that for masses near $2 m_{\pi}$, the contributions from $\pi^{0}$ and $\eta$ Dalitz decays are two or three orders of magnitude above the $q \bar{q}$ annihilation spectrum. However, it has been shown for zero $p_{T}$ dileptons having small invariant masses, that the perturbative corrections can be several orders of magnitude larger than the Born term [8]. So, we consider this $q \bar{q}$ annihilation spectrum as a lower limit only.

The contribution to the invariant mass spectrum from pion scattering with virtual bremsstrahlung shown in Ref. [7] is the same order as the $\pi^{0}$ and $\eta$ Dalitz decays in the region $M \leq 2 m_{\pi}$. However, the authors considered only the processes $\pi^{ \pm} \pi^{0} \rightarrow \pi^{ \pm} \pi^{0} \gamma^{*}$. Our purpose here is to supplement their results by generalizing the formulae to include other pion-pion reactions, such as $\pi^{ \pm} \pi^{\mp} \rightarrow \pi^{ \pm} \pi^{\mp} \gamma^{*}$. Contributions from $\pi^{ \pm} \pi^{ \pm} \rightarrow \pi^{ \pm} \pi^{ \pm} \gamma^{*}$ are ignored because one expects significant cancellation from like-charge interference [9]. Note the important difference in the ordering of the charges. Furthermore, we use these generalizations along with lattice gauge theory data on screening to estimate pair production from quark-quark and quark-gluon scattering processes within a soft photon approximation [10].

We imagine the following picture for $e^{+} e^{-}$production through virtual bremsstrahlung in thermalized hadronic matter. If the initial temperature of the matter is $T_{i}>T_{c}=200$ $\mathrm{MeV}$, then virtual bremsstrahlung from quarks occurs. As the system evolves in time it cools. If the QGP-hadron phase transition is first order at $T=T_{c}$ the mixed phase may be realized. In this state there will be dilepton emission from both quarks and pions. Then, for temperatures lower than $T_{c}$, say $T_{c}>T>T_{f} \simeq 140 \mathrm{MeV}$, one need only consider radiation from pions. Let us now estimate the rates of $e^{+} e^{-}$production in the quark and pion phases in the pursuit of a time-evolution integrated pair production mass spectrum.

In the reaction $\pi \pi \rightarrow \pi \pi$ one expects radiation of real and virtual photons as depicted in Fig. 1, with the external lines being pions and the shaded region representing the strong interaction. In the soft photon approximation [11], to which we restrict our discussion, only the initial and final states radiate virtual quanta. In this approximation, the differential cross section for $e^{+} e^{-}$pair production is

$$
\begin{aligned}
\frac{d \sigma_{\pi \pi}^{e^{+} e^{-}}}{d M^{2}}= & \frac{\alpha^{2}}{8 \pi^{4}} \frac{1}{M^{2}} \int|\epsilon \cdot J|^{2} \frac{d \sigma_{\pi \pi}}{d t} \\
& \times \delta\left(M^{2}-\left(p_{+}+p_{-}\right)^{2}\right) \frac{d^{3} p_{+} d^{3} p_{-}}{E_{+} E_{-}} d t
\end{aligned}
$$

where $t$ is the four-momentum transfer in the $\pi \pi$ collision, $M$ is the invariant mass of the $e^{+} e^{-}$pair, $p_{+}$and $p_{-}$are the momenta of the positron and electron respectively, and

$$
|\epsilon \cdot J|^{2}=\sum_{\delta} \epsilon_{\delta} \cdot J \epsilon_{\delta} \cdot J
$$

The sum is taken over all photon polarizations, and the current $J$ in the reaction $\pi_{a} \pi_{b} \rightarrow \pi_{c} \pi_{d}$ is

$$
J^{\mu}=-\widehat{Q}_{a} \frac{p_{a}^{\mu}}{p_{a} \cdot q}-\widehat{Q}_{b} \frac{p_{b}^{\mu}}{p_{b} \cdot q}+\widehat{Q}_{c} \frac{p_{c}^{\mu}}{p_{c} \cdot q}+\widehat{Q}_{d} \frac{p_{d}^{\mu}}{p_{d} \cdot q}
$$

where $\widehat{Q}_{i} \equiv Q_{\pi_{i}} /|e|$ and $Q_{\pi_{i}}$ is the charge of the $i$ th pion. When one of the incoming pions is electrically neutral, Eq. (0.2) can be approximated for $|t|<4 m_{\pi}^{2}$ by [1]] 


$$
|\epsilon \cdot J|^{2}=\frac{2}{3} \frac{1}{q_{0}^{2}}\left(\frac{-t}{m_{\pi}^{2}}\right) .
$$

The square of the photon's energy, $q_{0}^{2}$, is then replaced by the symmetrized combination $E\left(E^{2}-M^{2}\right)^{1 / 2}$, with $E=E_{+}+E_{-}=\left|\vec{p}_{+}\right|+\left|\vec{p}_{-}\right|$. Within the same region of validity, namely, $|t|<4 m_{\pi}^{2}$, we calculate $|\epsilon \cdot J|^{2}$ for all possible charge combinations in $\pi_{a} \pi_{b} \rightarrow \pi_{a} \pi_{b}$ $\left(\pi^{ \pm} \pi^{0} \rightarrow \pi^{ \pm} \pi^{0}\right.$ and $\left.\pi^{ \pm} \pi^{\mp} \rightarrow \pi^{ \pm} \pi^{\mp}\right)$ to be

$$
|\epsilon \cdot J|^{2}=\frac{2}{3} \frac{1}{q_{0}^{2}}\left(\frac{-t}{m_{\pi}^{2}}\right)\left[\left(\widehat{Q}_{a}^{2}+\widehat{Q}_{b}^{2}\right)-\frac{3}{2} \widehat{Q}_{a} \widehat{Q}_{b} f(s)\right],
$$

where

$$
\begin{aligned}
f(s)= & \frac{s}{2\left(s-4 m_{\pi}^{2}\right)}-\frac{s-4 m_{\pi}^{2}}{2 s} \\
& -\frac{m_{\pi}^{2}}{s}\left\{\frac{2 \sqrt{s}}{\sqrt{s-4 m_{\pi}^{2}}}+\left(\frac{\sqrt{s}}{\sqrt{s-4 m_{\pi}^{2}}}\right)^{3}\right. \\
& \left.+\frac{\sqrt{s-4 m_{\pi}^{2}}}{\sqrt{s}}\right\} \ln \left|\frac{\sqrt{s}+\sqrt{s-4 m_{\pi}^{2}}}{\sqrt{s}-\sqrt{s-4 m_{\pi}^{2}}}\right| .
\end{aligned}
$$

The interference function $f(s)$ behaves in the following manner. In the limit $\sqrt{s} \rightarrow 2 m_{\pi}$, $f(s) \rightarrow-2 / 3$ and as $\sqrt{s} \rightarrow \infty, f(s) \rightarrow 0$. In the small $\sqrt{s}$ region $\left(\sqrt{s} \rightarrow 2 m_{\pi}\right)$ the factor $|\epsilon \cdot J|_{\pi^{ \pm} \pi^{\mp}}^{2}=|\epsilon \cdot J|_{\pi^{ \pm} \pi^{0}}^{2}$, but for $\sqrt{s} \rightarrow \infty,|\epsilon \cdot J|_{\pi^{ \pm} \pi^{\mp}}^{2}=2|\epsilon \cdot J|_{\pi^{ \pm} \pi^{0}}^{2}$.

The momentum integration in Eq. (0.1) gives

$$
\begin{aligned}
\frac{d \sigma_{\pi \pi}^{e^{+} e^{-}}}{d M^{2}}= & \frac{\alpha^{2}}{3 \pi^{2}} \frac{\bar{\sigma}(s)}{M^{2}} \ln \left[\frac{\sqrt{s}-2 m_{\pi}}{M}\right] \\
& \times\left\{\left(\widehat{Q}_{a}^{2}+\widehat{Q}_{b}^{2}\right)-\frac{3}{2} \widehat{Q}_{a} \widehat{Q}_{b} f(s)\right\},
\end{aligned}
$$

where $\bar{\sigma}(s)$ is the momentum transfer weighted cross section. If $d \sigma / d t$ is a symmetric function of $t$ and $u$, then

$$
\bar{\sigma}(s)=2 \sigma_{e l}(s)\left[\frac{s}{4 m_{\pi}^{2}}-1\right] .
$$

The elastic pion-pion cross section is parametrized the same as in Ref. [7]. For $\sqrt{s} \leq 0.6 \mathrm{GeV}$ the chiral model expression is adopted, the $\rho$-resonance form of $\sigma_{e l}$ is used for $0.6<\sqrt{s} \leq 1.5$ $\mathrm{GeV}$ and finally, a constant value of $5 \mathrm{mb}$ is taken for $\sqrt{s}>1.5 \mathrm{GeV}$. The $e^{+} e^{-}$production rate through virtual bremsstrahlung from pions is [11]

$$
\begin{aligned}
\frac{d N}{d^{4} x d M^{2}}= & \frac{T^{6}}{(2 \pi)^{4}} \int_{z_{\min } / T}^{\infty} d z z^{2} \\
& \times\left(z^{2}-\frac{4 m_{\pi}^{2}}{T^{2}}\right) \mathcal{K}_{1}(z) \frac{d \sigma_{\pi \pi}^{e^{+} e^{-}}}{d M^{2}}
\end{aligned}
$$


where $z_{\text {min }}=2 m_{\pi}+M, d^{4} x$ is an element of four-volume of the hadronic matter, and $\mathcal{K}_{1}$ is the modified Bessel function. In Fig. 目 we show the rate of $e^{+} e^{-}$production from the sum of $\pi^{ \pm} \pi^{0}$ and $\pi^{ \pm} \pi^{\mp}$ scattering at a temperature $T=200 \mathrm{MeV}$. Also in Fig. 2 we compare our calculations with the rate of production using the chiral model with quartic pion interactions $\mathcal{L}_{I}=\lambda(\vec{\pi} \cdot \vec{\pi})^{2} / 4(\lambda=1.4$ from $\pi \pi$ scattering lengths) [12]. Our results reproduce the rate from the chiral model in the low mass region $M<100 \mathrm{MeV}$ only if $\sqrt{s}$ is restricted to the non-resonance region, say $\sqrt{s} \leq 0.48 \mathrm{GeV}$. Electron-positron pairs with invariant masses larger than $\sim 100 \mathrm{MeV}$ are attributed to $\sqrt{s}$ in the resonance region and beyond.

The soft photon approximation has the advantage that the electromagnetic and strong interaction components of the matrix elements disentangle leaving separate multiplicative factors (Low's theorem). We utilize this property again to simplify the contributions to the $e^{+} e^{-}$spectrum from quark-quark and quark-gluon scattering. The same collection of diagrams from Fig. 1 are considered with the charged external lines being $u$ or $d$ quarks (or antiquarks) and neutral external lines being gluons. In total we include six quark-quark (or antiquark) and four quark (or antiquark)-gluon diagrams. The electromagnetic amplitude is obtained from Eq. (0.5) by replacing the pion mass with the quark mass and by setting $\widehat{Q}_{a}$ and $\widehat{Q}_{b}$ to the appropriate quark electric charges. The strong interaction cross sections $d \sigma_{q g} / d t$ and $d \sigma_{q q} / d t$ are well known in the perturbative vacuum at the one-gluon-exchange level to be 13

$$
\frac{d \sigma_{a b}}{d t}=\frac{C_{a b} 2 \pi \alpha_{s}^{2}}{t^{2}}
$$

where

$$
C_{a b}=\left\{\begin{array}{l}
1(q g \rightarrow q g) \\
\frac{4}{9}(q q \rightarrow q q)
\end{array} .\right.
$$

For hot hadronic matter this is clearly inadequate. At finite temperatures, static colorelectric fields are shielded by quarks and gluons, and color-magnetic fields are shielded by non-perturbative effects [14]. To include such effects in a finite-temperature evaluation of diagrams like the one in Fig. 1, we use the color-electric mass $m_{E}^{2}=6 \pi \alpha_{s}(t) T^{2}$, and the lattice gauge result for the color-magnetic mass $m_{M}^{2}=25 \alpha_{s}^{2}(t) T^{2}$ [15] of the exchanged gluon. Then for massless quarks whose three-momenta are initially anticollinear in the frame fixed by the finite-temperature medium, we have [14]

$$
\frac{d \sigma_{a b}}{d t}=C_{a b} \frac{\pi}{2} \frac{\alpha_{s}^{2}(t)\left(2 t-m_{E}^{2}-m_{M}^{2}\right)^{2}}{\left(t-m_{E}^{2}\right)^{2}\left(t-m_{M}^{2}\right)^{2}}
$$

We check the validity of using massless quarks by assuming $\alpha_{E}(t) \simeq \alpha_{M}(t)$ and introducing finite quark masses. The dilepton production rates change very little, so to this level of accuracy, Eq. (0.12) is acceptable. Note that in the limit $m_{E}, m_{M} \rightarrow 0$ (free space) the resulting cross section reproduces Eq. (0.10). Realistically, $\alpha_{s}$ is also temperature dependent owing to many-body effects. Since we make small $|t|$ approximations throughout, we ignore the momentum dependence and adopt the lattice gauge theory renormalization group result for the temperature dependence [16]

$$
\alpha_{s}(T)=\frac{6 \pi}{\left(33-2 N_{f}\right) \ln \left(a T / T_{c}\right)},
$$


with $a \sim 8$.

Having the strong interaction cross sections, we can use Eq. (0.9) to calculate the rate of $e^{+} e^{-}$production through virtual bremsstrahlung in $q q \rightarrow q q$ scattering by replacing the pion mass with the quark mass and multiplying by the appropriate spin degeneracy factor 4. For $q g \rightarrow q g$ scattering we must use

$$
\begin{aligned}
\frac{d N}{d^{4} x d M^{2}}= & \frac{T^{6}}{(2 \pi)^{4}} \int_{z_{\min } / T}^{\infty} d z \\
& \times\left(z^{2}-\frac{m_{q}^{2}}{T^{2}}\right)^{2} \mathcal{K}_{1}(z) \frac{d \sigma_{q g}^{e^{+} e^{-}}}{d M^{2}}
\end{aligned}
$$

where this time $z_{\text {min }}=m_{q}+M$ and the spin degeneracy to multiply Eq. (0.14) is 6. Kinematics also changes the argument of the logarithm in Eq. (0.7) to $\left(\sqrt{s}-m_{q}\right) / M$.

A choice must be made for the quark mass to be used in Eqs. (0.5 0.9) and (0.14). Chiral invariance is restored for temperatures near the phase transition, so constituent quarks become current quarks with masses $m_{q} \simeq 5 \mathrm{MeV}$. On the another hand, in a QGP the fermion mass induced by many-body effects is [2, 3] $m_{q}=\left(2 \pi \alpha_{s} / 3\right)^{1 / 2} T$, which is much greater than $5 \mathrm{MeV}$. Since the quark-mass dependence in the rate is roughly $1 / m_{q}^{2}$ due to Eq. (0.8) and the temperature dependence is roughly $T^{6}$ as in Eq. (0.9), an upper limit on the rate is obtained using $m_{q}=5 \mathrm{MeV}$ at $T=300 \mathrm{MeV}$. Similarly, a lower limit on the rate is obtained by using $m_{q}=300 \mathrm{MeV}$ at the phase transition temperature. We show in Fig. 3 these lower and upper limits on the quark-driven rate, the rate obtained using the induced quark mass at two different values of temperature, and finally, the pion-driven contribution at $T_{c}$. From this one may conclude that the pion gas is slightly more luminous than our medium quark gas at $T_{c}$, for the range of invariant masses we consider. The rate of production from the current quarks is much larger than the pion rate.

Total $e^{+} e^{-}$production from competing quark and pion processes is obtained only after integrating the rate from Eq. (0.9) over the space-time history of the nuclear system. Using the standard 1+1 dimensional expansion in a Bjorken picture 17, 18, we reproduce the $\pi^{ \pm} \pi^{0}$ results of Ref. [7] from mixed plus cooling phases using their values for the relevant temperatures. By including the $\pi^{ \pm} \pi^{\mp}$ reactions we find a $20 \%$ enhancement over their result. The enhancement is smaller than one would expect, but in our case the cross section is $\sigma_{e l} \sim \sigma^{\pi^{+} \pi^{0}}+\sigma^{\pi^{-} \pi^{0}}+\sigma^{\pi^{+} \pi^{-}}$with an equal weighting $1 / 3$ of each channel, which is different from Ref. [7] where $\sigma_{e l} \sim \sigma^{\pi^{+} \pi^{0}}+\sigma^{\pi^{-} \pi^{0}}$ and therefore, the weighting is $1 / 2$ for both channels. We go on to choose temperatures $T_{i}=300 \mathrm{MeV}, T_{c}=200 \mathrm{MeV}$, and $T_{f}=140$ $\mathrm{MeV}$ in order to investigate other conditions likely to be found in heavy ion collisions at RHIC and LHC energies. In Fig. 1 we present the resulting invariant mass spectra through virtual bremsstrahlung from quarks and pions separately. The quark result is the sum of contributions from the cooling $\left(T_{i}>T>T_{c}\right)$ plus mixed $\left(T=T_{c}\right)$ phases and the pion result is the sum of contributions from the mixed $\left(T=T_{c}\right)$ plus cooling $\left(T_{c}>T>T_{f}\right)$ phases. The pion cooling-phase contribution is the larger contributor to its final spectrum; whereas the mixed-phase dominates the resulting quark spectrum. For a more complete comparison with other competing processes, we show also the results from $\pi^{0}$ and $\eta$ Dalitz decay as well as $q \bar{q} \rightarrow \gamma^{*} \rightarrow e^{+} e^{-}$annihilation in the Born approximation. Using our values of $T_{i}, T_{c}$ and $T_{f}$, the medium quark contribution (from cooling plus mixed phases) and the constituent quark 
contribution (from the mixed phase only) are both lower than the $\pi^{0}$ Dalitz, the $\eta$ Dalitz, and well below the pion contribution. On the other hand, the current quark contribution is larger than $\pi^{0}$ or $\eta$ Dalitz; it is even larger than the resulting pion contribution by a factor of 10 or more. However, we believe the true quark contribution will be nearer to the medium quark result. This being true, virtual bremsstrahlung from pions will be the largest source of low-mass $e^{+} e^{-}$pairs. This points out the need for more elaborate calculations, perhaps based on nonequilibrium approaches.

Assuming only soft virtual photons greatly simplified our task by eliminating the need to exactly evaluate the many Feynman diagrams that contribute to $e^{+} e^{-}$production. But the validity of this popular approach may be questioned. For instance, the restriction that the momentum transfer $|t|$ be less than $4 m_{\pi}^{2}$ for pions and less than $4 m_{q}^{2}$ for the quarks is arguably too restrictive. Also, many-body effects introduce temperature dependences into the meson masses, widths and coupling constants and therefore, into the $\pi \pi$ elastic cross section likely to be quite strong near $T_{c}$. Detailed discussions of these effects will be presented in a future paper [19].

\section{ACKNOWLEDGMENTS}

One of us (V.E.) is indebted to Profs. S. K. Mark and P. Depommier for helping organize his stay at McGill University. This work was supported by the National Science and Engineering Research Council of Canada and by the FCAR fund of the Québec Government. 


\section{REFERENCES}

* Permanent address: Department of High Energy Physics, Moscow Physical Engineering Institute, Kashirskoe Shosse 31, 115409, Moscow, Russia.

[1] E. Feinberg, Nuov. Cim. A 34, 39 (1976).

[2] E. Shuryak, Phys. Lett. B 79, 135 (1978).

[3] E. Shuryak, Phys. Rep. 67, 71 (1980).

[4] A. Shor, Phys. Lett. B 233, 231 (1989).

[5] K. Eskola and J. Lindfors, Z. Phys. C 46, 141 (1990).

[6] M. Asakawa and T. Matsui, Phys. Rev. D 43, 2871 (1991).

[7] J. Cleymans, K. Redlich and H. Satz, Z. Phys.C 53, 517 (1991).

[8] E. Braaten, R. D. Pisarski and T. Chiang Yuan, Phys. Rev. Lett. 64, 2242 (1990).

[9] K. L. Haglin, Ann. Phys. (N.Y.), 212, 84 (1991).

[10] R. Ruckl, Phys. Lett. B 64, 39 (1976).

[11] C. Gale and J. Kapusta, Phys. Rev. C 35, 2107 (1987).

[12] A. Weldon, Phys. Rev. Lett. 66, 293 (1991).

[13] J. Leader and E. Predazzi, An Introduction to Gauge Theories and the "New Physics", Cambridge Univ. Press., 1982.

[14] P. Danielewicz and M. Gyulassy, Phys. Rev. D 31, 53 (1985).

[15] T. DeGrand and D. Toussaint, Phys. Rev. D 25, 526 (1982).

[16] F. Karsch, Z. Phys. C 38, 147 (1988).

[17] J. Bjorken, Phys. Rev. D 27, 140 (1983).

[18] K. Kajantie et al., Phys. Rev. D 34, 2746 (1986).

[19] K. Haglin, C. Gale and V. Emel'yanov (in preparation). 


\section{FIGURES}

FIG. 1. Lepton pair production through virtual bremsstrahlung in scattering of particles $a b \rightarrow c d$. The particles might be charged or uncharged pions, quarks or antiquarks or gluons. The shaded region indicates a strong interaction.

FIG. 2. Chiral model predictions for the $e^{+} e^{-}$production rate as compared with our calculation. The dashed lines are our estimations with varying values for the upper limit on the energy and the solid line is from the chiral model.

FIG. 3. Mass dependence of quark-driven and pion-driven production rates at temperatures $T=200 \mathrm{MeV}$ and $T=300 \mathrm{MeV}$.

FIG. 4. Dilepton mass spectra for LHC energies: The solid curve is the total contribution from pion scattering processes, the dot-dashed curves result from quark-quark and quark-gluon

scattering with different quark masses, the long-dashed curve is the result of $q \bar{q}$ annihilation in a Born approximation, and finally, the short-dashed curves are Dalitz decay spectra. 